\title{
An Investigation into Training of Elementary and Secondary Level Teachers Regarding
} Presentation Skills

\author{
* Dr. Muhammad Malik, Assistant Professor (Corresponding Author) \\ ** Dr. Muhammad Naeemullah, Associate Professor \\ *** Mr. Wahid Mehmood, Scholar
}

\begin{abstract}
This research aimed to measure the drawbacks of in-service training Design for teachers at elementary and secondary levels in Punjab. The main objective of the study was to investigate whether training related to presentation skills is given to teachers or not. The population of the study consisted of all Trainers and Trainees (Teachers) at Elementary and Secondary level in Punjab. The sample of the study was comprised of 665 trainers and trainees and the sample for supplementary Questioners comprised of 621 trainers and trainees. Data was collected through Questioners. Two separate Questioners were developed for trainees and trainers and a supplementary Questionnaire was also prepared to address the remaining aspects related to disaster management, Computer skills, use of social media, and multigrade teaching. Three-Point scale was used for questionnaires and weightage was given as (1) Mostly (2) To Some Extent (3) Not at All. Chi-Square test was applied for Statistical analysis. The researcher personally visited the schools and distributed questionnaires among respondents. Data collected through research instruments were tabulated, analyzed, and was discussed. Rawalpindi and Gujranwala Divisions were selected for collection of data and the study was delimited to District Attock, Rawalpindi, and Gujranwala. This Research is fruitful for trainees and trainers as well as the administration of the Training Institutes. In the end, the Findings of the research show satisfactory results but most of the areas were neglected which needs to be addressed.
\end{abstract}

Keywords: HRM, HRD, Training, Presentation Skills.

Introduction

According to DeCenzo, and Robbins (2010), Human Resource has a concern about the measurement of people management and its dimension about the management skills. It is fundamental of all organizations that it depends upon the people as well as their skills and managerial powers. Every organization depends upon the skilled workers as well as their well-trained services. There are many types of organizations like business, government, health, restoration, and societal action. There are many functions of HRM and HRD works under the supervision of HRM. HRD is a structure that helps the employees to get their skills, enhance their ability, and to get knowledge. It is framework to help the employees. It is systematic activities to help employees to enhance their skills and development to meet the futuristic goals (Akbar, 2001). HRD has many activities like Training and Development in education and to enhance job opportunities in a society. Teachers are the backbone of a society. It is considered a natural phenomenon that well-trained teachers are responsible for the progress of a country as well as societal activities. It is necessary to give proper training to teachers as they are the nation builders. Training is very essential for teachers. The education extension center is developed by the Government of Punjab in 1959 and it is given the name of Directorate of Staff Development (DSD). DSD is responsible for teachers training and for conducting trainings for the betterment of teachers and the enhancement of their teaching skills. It is situated in Lahore as well monitored from Lahore. DSD has the following classification.

\section{District Training and Support Centre (DTSC)}

This Centre operates in Districts.

\footnotetext{
* Northern University, Nowshera, Khyber Pakhtunkhwa, Pakistan Email: malik@ northern.edu.pk

** Northern University, Nowshera, Khyber Pakhtunkhwa, Pakistan

*** Northern University, Nowshera, Khyber Pakhtunkhwa, Pakistan
} 


\section{Cluster Training and Support Centre (CTSC}

15-30 schools collectively constitute CTSC. Supervision and Finance to Cluster Training and Support Centre is provided by District Training and Support Centers. There is a total of 36 DTSC and 1992 CTSC in Punjab. Total Trainees at Elementary and Secondary level in Punjab are 321064 which are further divided into 155824 Male and 165240 Females. The total Trainer in Punjab is 3385 and the ratio of Trainers to Trainee is 1:95. DTSC Attock has 63 CTSC. There are 3431 Male and 4345 Female Trainees. The Trainers are 96 Male and 14 Female. DTSC Rawalpindi has 76 Cluster Training and Support Centre (CTSC). There are 5296 males and 9193 female trainees. The trainers are 115 males and 25 females. DTSC Gujranwala has 65 CTSC. There are 5796 males and 6360 female trainees. The Trainers are 124 (Retrieved from http://www.dsd.edu.pk.). Now the name of DSD has been changed to Quaid-e-Azam Academy for Educational Development (QAED).

\section{Statement of the problem}

It is needed to investigate whether the presentation skills are inculcated in the training of elementary and secondary level teachers or not.

\section{Objective of the study}

The main objective of the study was to investigate whether training related to presentation skills is given to teachers or not.

\section{Significance of the study}

This study suggests that which types of skills should be inculcated in teachers so that they became modern teachers and fruitful for the betterment of Pakistani society. This study is beneficial for trainers, trainees as well as Govt. of Pakistan Education Sector.

\section{Method and Procedure}

\section{Population}

All trainers and trainees at the Elementary and secondary level constitute the population of the study. Punjab has 36 DTSC and 1992 CTSC. Total trainees at elementary and secondary levels in Punjab are 321064 which are further divided into 155824 male and 165240 females. The total trainer in Punjab was 3385 and the ratio of trainers to trainee was 1:95.

\section{Sample}

The sample of the study consisted of 665 trainer and trainee and induction trainee.

\begin{tabular}{cccc}
\hline Category & Male & Female & Total \\
\hline Trainees & 163 & 11 & 374 \\
Trainer & 98 & 35 & 133 \\
Induction Trainee & 79 & 79 & 158 \\
Grand Total & & & 665 \\
\hline
\end{tabular}

The sample of the study for the supplementary questionnaire was

\begin{tabular}{cccc}
\hline Category & Male & Female & Total \\
\hline Trainees & 139 & 316 & 455 \\
Trainer & 67 & 22 & 89 \\
Induction Trainee & 0 & 77 & 77 \\
Grand Total & & & 621 \\
\hline
\end{tabular}

\section{Research Instrument}

Data was collected through Questioners. Two separate Questioners were developed for trainees and trainers and a supplementary Questionnaire was also prepared to address the remaining aspects related to disaster management, Computer skills, use of social media, and multigrade teaching. Three-Point scale was used for questionnaires and weight-age was given as (1) Mostly (2) To Some Extent (3) Not at All

\section{Delimitation of the Study}

The study was delimited to District Attock, Rawalpindi, and Gujranwala for the collection of Data.

Data Collection

Data was collected from District Rawalpindi, Attock, and Gujranwala. The researcher personally visited the schools and distributed questionnaires among respondents.

\section{Analysis of Data}

Data were analyzed by using the Chi-square test and were tabulated. 


\section{Review of Related Literature}

Education is very essential for the progress of a society as well as for the development of a country. It plays a very vital and basic role in the development of the nation. A teacher is a backbone and nation builders of the society. They play a very basic and essential role in the development of the country. It is very essential to develop a teacher's ability and to give them modern means of training so that they can train their students to meet the modern trends and education.

According to Belka (2002), a teacher must be very cleared about his task as well as his content. In this way, he can give a better understanding to students and can achieve his goals. It is a fundamental phenomenon that one must very clear about one's concept in this way he can give a better understanding to listens. Presentation skills are a set of strategies that enable the individual to contact with audience and deliver his message very clearly. So, Teachers face many problems during his lectures like discipline, non-attention of students, lack of inspiration, and many other hurdles and inconvenience. These problems create an attention gap between students and teachers. For tackling these problems teachers must be equipped with presentation skills. It is natural phenomena that speech is only one-sided, and the teacher forgets about the importance of nonverbal communication as well as body language and extra linguistics features. There are two aspects of nonverbal communication like the connection of eyes and body expressions. Both are very important to convey a message. It is a natural law that sometimes body language and facial expressions say more than words. Through these teachers can convey messages to the students and audience as well as listeners. While most of the classroom spend its time with eyes firmly fixed on the books, the boards, the overhead projector, rounding, and walking around the window during teaching, listening, and learning environment (Ledbury, White \& Darn, 2004). Jing (2006) claimed "that too much eye contact is bad. Eyes contact is related to hope and with trust, good feelings and understanding all of which are important and better qualities". The teacher must be careful about his eye interaction while he wants to give attention and praise to his students in the classroom. Language is a gateway to knowledge. It is a source of communication as well as a medium of communication. Through language, a teacher can convey his message to his students. Language is a medium to convey a message. The means of instruction is a very typical issue, especially in Pakistan. It is a very controversial issue in Pakistan because in our society there are many mediums of instructions are used (Ahmed, 2011).

\section{Analysis and Interpretation of Data}

Chi-square test is used for the analysis and description of data.

Table 1. Trainees are trained in the proper use of the Writing Board.

\begin{tabular}{cccccccccc}
\hline Respondents & Category & $\mathbf{N}$ & Mostly & \%age & $\begin{array}{c}\text { To some } \\
\text { extent }\end{array}$ & \%age & $\begin{array}{c}\text { Not } \\
\text { at all }\end{array}$ & \%age & $\boldsymbol{\chi}^{\mathbf{2}}$ \\
\hline Trainer & Male & 98 & 58 & 59.18 & 38 & 38.77 & 2 & 2.04 & 48.80 \\
& Female & 35 & 21 & 60 & 13 & 37.14 & 1 & 2.85 & 16.91 \\
Trainee & Male & 163 & 123 & 75.46 & 37 & 22.69 & 3 & 1.84 & 141.67 \\
& Female & 211 & 154 & 72.98 & 50 & 23.69 & 7 & 3.31 & 163.21 \\
Induction & Male & 79 & 39 & 49.36 & 33 & 41.77 & 7 & 8.86 & 22.26 \\
Trainee & Female & 79 & 43 & 54.43 & 24 & 30.37 & 12 & 15.18 & 18.79 \\
Total & & $\mathbf{6 6 5}$ & $\mathbf{4 3 8}$ & & $\mathbf{1 9 5}$ & & $\mathbf{3 2}$ & & \\
\hline
\end{tabular}

Degree of freedom $=2$, Significance level $=0.05$, Table Value $=5.991$

According to table 1, the value of chi -Square of trainers of males is 48.80 but it is greater than the table value. Hence the statement is accepted, and the result is found significant through this table. According to Chi-Square, the value of female trainees is 16.91 which is greater than the table value. Therefore, the result is in favor of the statement and the statement is accepted. According to ChiSquare, the value of males is 141.67 and it is greater than the table value. Therefore, result is found significant and the value has been accepted accordingly. The value of female trainees according to Chi-Square is 163.21 and it is greater than the table value, while the result is significant, and it is accepted. According to Chi-Square, the value of the induction trainee is 22.96 which is greater than the table value. Therefore, it is found significant and the statement is accepted accordingly while the female induction trainee value is 18.79 which is greater than the table value while the result is found significant and it is favor of the statement and has accepted. 
Table 2. Concept "Use of Eye contact during class" is inculcated during Training

\begin{tabular}{cccccccccc}
\hline Respondents & Category & $\mathbf{N}$ & Mostly & $\mathbf{\% a g e}$ & $\begin{array}{c}\text { To some } \\
\text { extent }\end{array}$ & \%age & $\begin{array}{c}\text { Not at } \\
\text { all }\end{array}$ & \%age & $\chi^{\mathbf{2}}$ \\
\hline \multirow{2}{*}{ Trainer } & Male & 98 & $\mathbf{4 2}$ & 42.85 & 43 & 43.87 & 13 & 13.26 & 17.60 \\
& Female & 35 & $\mathbf{2 2}$ & 62.85 & 13 & 37.14 & 0 & 0 & 20.41 \\
Trainee & Male & 163 & $\mathbf{1 0 3}$ & 63.19 & 49 & 30.06 & 11 & 6.74 & 79.16 \\
Induction & Female & 211 & $\mathbf{1 1 8}$ & 55.92 & 72 & 34.12 & 21 & 9.95 & 67.26 \\
Trainee & Male & 79 & $\mathbf{3 4}$ & 43.03 & 32 & 40.50 & 13 & 16.45 & 10.34 \\
Total & & $\mathbf{6 6 5}$ & $\mathbf{3 6 4}$ & 56.96 & 18 & 22.78 & 16 & 20.25 & 20.18 \\
\hline
\end{tabular}

Degree of freedom $=2$, Significance level $=0.05$, Table Value $=5.991$

In this table, the value of the Chi-Square of Trainer Male is 17.60 which is greater than and value of female trainee is 20.41 which is greater than the table value results are found in favor of the statement and found significant and it is accepted. The value of the Chi-Square of Trainer Male is 79.16and the value of the female trainee is 67.26.therefore both values are greater than the table value while results are found in favor of the statement and found significant and it is accepted. In this value of ChiSquare of induction Trainer Male is 10.34 and the value of female induction trainee is 20.18.therefore both values are greater than the table value while results are found in favor of the statement and found significant and it is accepted.

Table 3. Trainees are trained in the proper Use of Medium of Instruction

\begin{tabular}{cccccccccc}
\hline Respondents & Category & $\mathbf{N}$ & Mostly & \%age & $\begin{array}{c}\text { To some } \\
\text { extent }\end{array}$ & \%age & Not at all & \%age & $\boldsymbol{\chi}^{\mathbf{2}}$ \\
\hline \multirow{2}{*}{ Trainer } & Male & 98 & 45 & 45,91 & 45 & 45,91 & 8 & 8.16 & 27.65 \\
& Female & 35 & 22 & 62.85 & 12 & 34.28 & 1 & 2.85 & 18.41 \\
Trainee & Male & 163 & 102 & 62.57 & 54 & 33.12 & 7 & 4.29 & 83.56 \\
Induction & Female & 211 & 121 & 57.34 & 82 & 38.86 & 8 & 3.79 & 94.11 \\
Trainee & Male & 79 & 41 & 51.89 & 33 & 41.77 & 5 & 6.32 & 27.49 \\
\multicolumn{1}{r}{ Total } & Female & 79 & 44 & 55.69 & 29 & 36.70 & 6 & 7.59 & 27.18 \\
\hline
\end{tabular}

Degree of freedom $=2$, Significance level $=0.05$, Table Value $=5.991$

It has been shown in this table that the value of the Chi-Square of Trainer Male is 27.65 and the value of female trainees is 18.41. therefore, both values are greater than the table value while results are found in favor of the statement and found significant and it is accepted. In table 3 the value of the ChiSquare of Trainer Male is 83.56 and the value of female trainee is 93.66.therefore both values are greater than the table value while results are found in favor of the statement and found significant and it is accepted. The value of the Chi-Square of induction Trainer Male is 27.49 and the value of female trainee is 27.18.therefore both values are greater than the table value while results are found in favor of the statement and found significant and it is accepted.

Table 4. Tips to gain confidence are given to trainees

\begin{tabular}{cccccccccc}
\hline Respondents & Category & $\mathbf{N}$ & Mostly & \%age & $\begin{array}{c}\text { To some } \\
\text { extent }\end{array}$ & \%age & Not at all & \%age & $\boldsymbol{\chi}^{\mathbf{2}}$ \\
\hline \multirow{2}{*}{ Trainer } & Male & 98 & 58 & 59.18 & 38 & 38.77 & 2 & 2.04 & 48.80 \\
& Female & 35 & 19 & 54.28 & 16 & 45.71 & 0 & 0 & 17.41 \\
Trainee & Male & 163 & 118 & 72.39 & 42 & 25.76 & 3 & 1.84 & 126.67 \\
Induction & Female & 211 & 158 & 74.88 & 49 & 23.22 & 4 & 1.89 & 179.14 \\
Trainee & Male & 79 & 60 & 75.94 & 14 & 17.72 & 5 & 6.32 & 66.95 \\
Total & & $\mathbf{6 6 5}$ & $\mathbf{4 7 0}$ & & $\mathbf{1 7 5}$ & & $\mathbf{2 0}$ & & 56.18 \\
\hline
\end{tabular}

Degree of freedom $=2$, Significance level $=0.05$, Table Value $=5.991$

It is represented above that value of the Chi-Square of Trainer Male is 48.80 which is greater than and value of female trainee is 17.41 which is less than the table value results are found in favor of the statement and found significant and it is accepted. The value of the Chi-Square of Trainer Male is 126.67 and the value of female trainee is 179.14 .therefore both values are greater than the table value while results are found in favor of the statement and found significant and it is accepted. The value of the Chi-Square of induction Trainer Male is 66.95 and the value of the female induction trainee is 
56.18.therefore both values are greater than the table value while results are found in favor of the statement and found significant and it is accepted.

Table 5. Subject Knowledge is focused during Training

\begin{tabular}{cccccccccc}
\hline Respondents & Category & $\mathbf{N}$ & Mostly & \%age & $\begin{array}{c}\text { To some } \\
\text { extent }\end{array}$ & \%age & $\begin{array}{c}\text { Not } \\
\text { at all }\end{array}$ & \%age & $\boldsymbol{\chi}^{\mathbf{2}}$ \\
\hline \multirow{2}{*}{ Trainer } & Male & 98 & 70 & 71.42 & 25 & 25.51 & 3 & 3.06 & 70.68 \\
& Female & 35 & 25 & 71.42 & 9 & 25.71 & 1 & 2.85 & 24.91 \\
Trainee & Male & 163 & 115 & 70.55 & 47 & 28.83 & 1 & 0.61 & 121.81 \\
& Female & 211 & 152 & 72.03 & 55 & 26.06 & 4 & 1.89 & 161.48 \\
Induction & Male & 79 & 48 & 60.75 & 25 & 31.64 & 6 & 7.59 & 34.02 \\
Trainee & Female & 79 & 45 & 56.96 & 27 & 34.17 & 7 & 8.86 & 27.79 \\
\multicolumn{1}{c}{ Total } & & $\mathbf{6 6 5}$ & $\mathbf{4 5 5}$ & & $\mathbf{1 8 8}$ & & $\mathbf{2 2}$ & & \\
\hline
\end{tabular}

Degree of freedom $=2$, Significance level $=0.05$, Table Value $=5.991$

No 5 has shown that the value of Chi-Square of Trainer Male is 70.68 which is greater than and value of female trainee is 24.91 which is greater than the table value results are found in favor of the statement and found significant and it is accepted. In Table 5 the value of the Chi-Square of Trainer Male is 121.81 and the value of female trainee is 161.48.therefore both values are greater than the table value while results are found in favor of the statement and found significant and it is accepted. It is given value of the Chi-Square of induction Trainer Male is 34.02and value of female induction trainee is 27.79.therefore both values are greater than the table value while results are found in favor of the statement and found significant and it is accepted.

Table 6. Trainees communication skills are strengthened during the Training

\begin{tabular}{|c|c|c|c|c|c|c|c|c|c|}
\hline Respondents & Category & $\mathbf{N}$ & Mostly & $\%$ age & $\begin{array}{c}\text { To } \\
\text { some } \\
\text { extent }\end{array}$ & $\%$ age & $\begin{array}{c}\text { Not } \\
\text { at all }\end{array}$ & $\%$ age & $\chi^{2}$ \\
\hline \multirow{2}{*}{ Trainer } & Male & 98 & 59 & 60.20 & 35 & 35.71 & 4 & 4.08 & 46.08 \\
\hline & Female & 35 & 25 & 71.42 & 9 & 25.71 & 1 & 2.85 & 24.91 \\
\hline \multirow{2}{*}{ Trainee } & Male & 163 & 122 & 74.84 & 39 & 23.92 & 2 & 1.22 & 139.85 \\
\hline & Female & 211 & 151 & 71.56 & 55 & 26.06 & 5 & 2.36 & 157.28 \\
\hline Induction & Male & 79 & 50 & 63.29 & 26 & 32.91 & 3 & 3.79 & 42.49 \\
\hline \multirow{2}{*}{$\begin{array}{l}\text { Trainee } \\
\text { Total }\end{array}$} & Female & 79 & 54 & 68.35 & 22 & 27.84 & 3 & 3.79 & 51.1 \\
\hline & & 665 & 461 & & 186 & & 18 & & \\
\hline
\end{tabular}

Degree of freedom $=2$, Significance level $=0.05$, Table Value $=5.991$

It is shown in 6 that the value of Chi-Square of Trainer Male is 46.08 which is greater than and value of female trainee is 24.91 which is greater than the table value results are found in favor of the statement and found significant and it is accepted. It is shown in table 6 that the value of the ChiSquare of Trainer Male is 139.85 and the value of female trainee is 157.28.therefore both values are greater than the table value while results are found in favor of the statement and found significant and it is accepted. The value of the Chi-Square of induction Trainer Male is 42.49 and the value of the female induction trainee is 51.1.therefore both values are greater than the table value while results are found in favor of the statement and found significant and it is accepted.

Table 7. Groups of Trainees are made, and they are given a task

\begin{tabular}{cccccccccc}
\hline Respondents & Category & $\mathbf{N}$ & Mostly & \%age & $\begin{array}{c}\text { To some } \\
\text { extent }\end{array}$ & \%age & $\begin{array}{c}\text { Not } \\
\text { at all }\end{array}$ & \%age & $\boldsymbol{\chi}^{\mathbf{2}}$ \\
\hline Trainer & Male & 98 & 63 & 64.28 & 34 & 34.69 & 1 & 1.02 & 58.33 \\
& Female & 35 & 15 & 42.85 & 20 & 57.14 & 0 & 0 & 18.08 \\
Trainee & Male & 163 & 128 & 78.52 & 34 & 20.85 & 1 & 0.61 & 160.81 \\
Induction & Female & 211 & 165 & 78.19 & 44 & 20.85 & 2 & 0.94 & 204.62 \\
Trainee & Male & 79 & 56 & 70.88 & 22 & 27.84 & 1 & 1.26 & 59.25 \\
Total & & $\mathbf{6 6 m}$ & $\mathbf{4 9 6}$ & & $\mathbf{1 6 4}$ & & $\mathbf{5}$ & & 106.95 \\
\hline
\end{tabular}

Degree of freedom $=2$, Significance level $=0.05$, Table Value $=5.991$

It is shown in 7 that the value of Chi-Square of Trainer Male is 58.33 which is greater than and value of female trainee is 18.08 which is less than the table value results are found in favor of the statement and found significant and it is accepted. It is shown in table 7 that the value of the Chi-Square of 
Trainer Male is 160.81 and the value of the female trainee is 204.62.therefore both values are greater than the table value while results are found in favor of the statement and found significant and it is accepted. Table 7 represents the value of the Chi-Square of induction Trainer Male is 59.25 and the value of the female induction trainee is 106.95.therefore both values are greater than the table value while results are found in favor of the statement and found significant and it is accepted.

Table 8. The Trainees Groups are asked to present their assignment in front of Other Trainees

\begin{tabular}{cccccccccc}
\hline \multirow{2}{*}{ Respondents } & Category & $\mathbf{N}$ & Mostly & \%age $\begin{array}{c}\text { To some } \\
\text { extent }\end{array}$ & \%age & $\begin{array}{c}\text { Not } \\
\text { at } \\
\text { all }\end{array}$ & \%age & $\boldsymbol{\chi}^{\mathbf{2}}$ \\
\hline \multirow{2}{*}{ Trainer } & Male & 98 & 50 & 51.02 & 43 & 43.87 & 5 & 5.10 & 35.53 \\
& Female & 35 & 16 & 45.71 & 19 & 54.28 & 0 & 0 & 17.41 \\
Trainee & Male & 163 & 117 & 71.77 & 44 & 26.99 & 2 & 1.22 & 125.42 \\
Induction & Female & 211 & 164 & 77.72 & 41 & 19.43 & 6 & 2.84 & 196.74 \\
Trainee & Male & 79 & 43 & 54.43 & 29 & 36.70 & 7 & 8.86 & 25.33 \\
\multicolumn{1}{r}{ Total } & Female & $\mathbf{7 9}$ & $\mathbf{5 2}$ & 65.82 & 21 & 26.58 & 6 & 7.59 & 42.34 \\
\hline
\end{tabular}

Degree of freedom $=2$, Significance level $=0.05$, Table Value $=5.991$

Table 8 represents that the value of the Chi-Square of Trainer Male is 35.53 which is greater than and value of female trainee is 17.41 which is less than the table value results are found in favor of the statement and found significant and it is accepted. It is shown in table 8 that the value of the ChiSquare of Trainer Male is 125.42 and the value of female trainee is 196.74.therefore both values are greater than the table value while results are found in favor of the statement and found significant and it is accepted. The value of the Chi-Square of induction Trainer Male is 25.33 and the value of the female induction trainee is 42.34.therefore both values are greater than the table value while results are found in favor of the statement and found significant and it is accepted.

Table 9 Training related to presentation skill is given

\begin{tabular}{cccccccccc}
\hline \multirow{2}{*}{ Respondents } & Category & $\mathbf{N}$ & Mostly & \%age & $\begin{array}{c}\text { To some } \\
\text { extent }\end{array}$ & \%age & $\begin{array}{c}\text { Not } \\
\text { at } \\
\text { all }\end{array}$ & \%age & $\boldsymbol{\chi}^{\mathbf{2}}$ \\
\hline \multirow{2}{*}{ Trainer } & Male & 98 & 50 & 51.02 & 42 & 42.85 & 6 & 6.12 & 33.29 \\
& Female & 35 & 22 & 62.85 & 12 & 34.28 & 1 & 2.85 & 18.41 \\
Trainee & Male & 163 & 113 & 69.32 & 44 & 26.99 & 6 & 3.68 & 108.97 \\
& Female & 211 & 168 & 79.62 & 37 & 17.53 & 6 & 2.84 & 211.26 \\
Induction & Male & 79 & 56 & 70.88 & 20 & 25.31 & 3 & 3.79 & 56.33 \\
Trainee & Female & $\mathbf{7 9}$ & $\mathbf{5 1}$ & $\mathbf{6 4 . 5 5}$ & 23 & 29.11 & 5 & 6.32 & 41.33 \\
\multicolumn{1}{c}{ Total } & & $\mathbf{6 6 5}$ & $\mathbf{4 6 0}$ & & $\mathbf{1 7 8}$ & & $\mathbf{2 7}$ & & \\
\hline
\end{tabular}

Degree of freedom $=2$, Significance level $=0.05$, Table Value $=5.991$

It is presented in the value of Chi-Square of Trainer Male is 33.29 which is greater than and value of female trainee is 18.41 which is greater than the table value results are found in favor of the statement and found significant and it is accepted. It is shown in table 9 that the value of the Chi-Square of Trainer Male is 108.97 and the value of female trainee is 211.26.therefore both values are greater than the table value while results are found in favor of the statement and found significant and it is accepted. The value of the Chi-Square of induction Trainer Male is 56.33 and the value of the female induction trainee is 41.33.therefore both values are greater than the table value while results are found in favor of the statement and found significant and it is accepted.

Table 10. Implementation of Brainstorming in the class is focused during Training

\begin{tabular}{cccccccccc}
\hline Respondents & Category & $\mathbf{N}$ & Mostly & \%age & $\begin{array}{c}\text { To some } \\
\text { extent }\end{array}$ & \%age & Not at all & \%age & $\boldsymbol{\chi}^{\mathbf{2}}$ \\
\hline Trainer & Male & 98 & 72 & 73.46 & 22 & 22.44 & 4 & 4.08 & 75.23 \\
& Female & 35 & 3 & 8.57 & 3 & 8.57 & 29 & 82.85 & 37.58 \\
Trainee & Male & 163 & 115 & 70.55 & 45 & 27.60 & 3 & 1.84 & 118.56 \\
& Female & 211 & 138 & 65.40 & 66 & 31.27 & 7 & 3.31 & 122.97 \\
Induction & Male & 79 & 48 & 60.75 & 24 & 30.37 & 7 & 8.86 & 32.64 \\
Trainee & Female & 79 & 48 & 60.75 & 29 & 36.70 & 2 & 2.53 & 41.1 \\
\multicolumn{1}{c}{ Total } & & $\mathbf{6 6 5}$ & $\mathbf{4 2 4}$ & & $\mathbf{1 8 9}$ & & $\mathbf{5 2}$ & & \\
\hline
\end{tabular}

Degree of freedom=2, Significance level $=0.05$, Table Value $=5.991$ 
Table 10 has represented that value of the Chi-Square of Trainer Male is 75.23 which is greater than and value of female trainee is 37.58 which is greater than the table value results are found in favor of the statement and found significant and it is accepted. It is shown in table 10 that the value of the ChiSquare of Trainer Male is 118.56 and the value of the female trainee is 122.97.therefore both values are greater than the table value while results are found in favor of the statement and found significant and it is accepted. The value of the Chi-Square of induction Trainer Male is 32.64 and the value of the female induction trainee is 41.1.therefore both values are greater than the table value while results are found in favor of the statement and found significant and it is accepted.

\section{Conclusions}

The researcher concluded the analysis of Data. The responses of Trainer, Trainee, and Induction Trainees are as given below:

1. It was concluded from the result that training by the Directorate of Staff Development, Imparts training to teachers related to proper use of writing Board.

2. Results show that the concept of the use of eye contact during class was inculcated in teachers but not at a satisfactory level.

3. The results of Table 3 show that training related to proper use of medium of instruction was given to some extent.

4. It was concluded that tips to gain confidence are given to teachers during training. It was also concluded that training related to focus on subject knowledge was given and the communication skills of teachers were strengthened during training. Further Groups of trainees are made, and they were given a task and trainees groups were asked to present their assignments in front of other trainees. Overall, it was concluded that training related to brainstorming and presentation skills was given.

\section{Recommendations}

The following recommendations were given based on derived Conclusions.

1. The findings of the study show that training related to "Concept of use of Eye Contact" was given but not at a satisfactory level. The use of eye contact has a vital role in communication without which one cannot understand that the audience is satisfied or not. It is recommended to give more attention to this aspect and during training, this concept should be inculcated in trainees.

2. In the teaching-learning process medium of instruction is just like the backbone without which learning is impossible. The findings of the study show that this aspect has been neglected during training at DSD therefore it needs to focus this aspect so that teachers may be trained properly for use of medium of instructions so that learning may become easy and understandable.

3. Findings show that training related to presentation skills was given ton trainees but it is strongly recommended to pay more attention to strengthening this skill in teachers so that they may teach students with confidence having strong subject knowledge and powerful

References communication skills.

Ahmed, S. I. (2011). Issue of medium of instruction in Pakistan. International Journal of social sciences and education, 1(1), 66-82.

Akbar, R.A. (2001). A study on Practice teaching on prospective secondary school Teachers and development of a practice teaching model, (Unpublished PhD dissertation) Rawalpindi, University Institute of Education and Research,

Belka, D. E. (2002). A strategy for improvement of learning presentations. Journal of Physical Education, Recreation \& Dance, 73(6), 32-35.

Coleman, H. (2010). Teaching and learning in Pakistan: The role of language in education. Islamabad: The British Council, 1-56.

DeCenzo, D. A., \& Robbins, S. P. (2010). Human Resource Management, International Student Version. John Wiley \& Sons.

Jing, H. (2006). Fostering learner autonomy within constraints: Negotiation and mediation in an atmosphere of collegiality.

Ledbury, R., White, I., \& Darn, S. (2004). The importance of eye contact in the classroom. The Internet TESL Journal, 10(8), 11-21. 
An Investigation into Training of Elementary and Secondary .... Malik, Naeemullah, Mehmood

Web References:

Directorate of Staff Development (2018). Retrieved from http://www.dsd.edu.pk.

Modern Teaching Skills (2018). Retrieved from https://www.examtime.com/blog/teaching-skills. 